\title{
BMJ Global Health Global impact of COVID-19 on newborn screening programmes
}

\author{
Vanesa Koracin, ${ }^{1} \mathrm{~J}$ Gerard Loeber, ${ }^{2}$ Matej Mlinaric, ${ }^{1}$ Tadej Battelino, ${ }^{1}$ \\ James R Bonham, ${ }^{3}$ Urh Groselj (i) , ${ }^{1}$ COVID-NBS ISNS global network
}

To cite: Koracin V, Loeber JG, Mlinaric M, et al. Global impact of COVID-19 on newborn screening programmes. BMJ Global Health 2022;7:e007780. doi:10.1136/ bmjgh-2021-007780

\section{Handling editor Stephanie M} Topp

- Additional supplemental material is published online only. To view, please visit the journal online (http://dx.doi.org/10. 1136/bmjgh-2021-007780)

VK and MM contributed equally.

Received 20 0ctober 2021 Accepted 20 January 2022

\section{Check for updates}

(c) Author(s) (or their employer(s)) 2022. Re-use permitted under CC BY-NC. No commercial re-use. See rights and permissions. Published by BMJ.

'Department of Endocrinology, Diabetes and Metabolism, UMC - University Children's Hospital Ljubljana; Faculty of Medicine, University of Ljubljana, Ljubljana, Slovenia International Society for Neonatal Screening (ISNS) Office, Bilthoven, The Netherlands

${ }^{3}$ Sheffield Children's Hospital NHS Foundation Trust, Sheffield, UK

Correspondence to Asst. Professor Urh Groselj; urh.groselj@kclj.si

\section{ABSTRACT}

Introduction The global COVID-19 pandemic has presented extraordinary disruption to healthcare services and exposed them to numerous challenges. Newborn screening (NBS) programmes were also affected; however, scarce data exist on the impact of COVID-19 on NBS.

Methods We conducted an international survey to assess the global impact of COVID-19 on NBS, with the main aim of gathering the experiences of the COVID-19 pandemic from a large and representative number of NBS centres worldwide.

Results The results of our study showed that COVID-19 impacted the NBS programmes, at least partially, in 29 out of 38 responding countries. Majority of the screening centres experienced a broad spectrum of difficulties and most were affected more in the second wave of the pandemic. Delays and unreliability with the postal service as well as flight cancellations caused delays in samples arriving to screening centres and with the provision of laboratory equipment and reagents. The availability of laboratory staff was sometimes reduced due to infection, quarantine or reassignment within the healthcare facility. Sample collection at home, second-tier tests and follow-up were also affected. Social restrictions and interruptions in public transport added to these difficulties. Only a limited number of centres managed to retain a fully functioning NBS programme.

Conclusion As the pandemic might continue or could recur in future years, it would be useful to develop guidelines to protect these valuable services.

\section{INTRODUCTION}

A global pandemic of COVID-19 caused by SARS-CoV-2 was announced by the WHO in March 2020. ${ }^{1}$ By 24 April 2021, 145216414 cases had been confirmed, including 3079390 people who died of the disease. ${ }^{2}$ In the first year of the pandemic, a two-wave pattern was observed in many countries, the first wave occurring in spring 2020, followed by the second in late summer and autumn. ${ }^{3}$ The reorganisation of healthcare systems towards the care of patients with COVID-19 infection then indirectly affected the management and treatment of non-infected patients with other chronic and rare diseases. ${ }^{4}$ These difficulties also affected screening facilities, follow-up

\section{Key questions}

What is already known?

- Newborn screening (NBS) programmes are important for early detection of inborn disorders and prevention of severe consequences on children's health.

- The COVID-19 pandemic has impacted healthcare systems worldwide at all levels.

What are the new findings?

- NBS was also affected by the pandemic in most of the responding countries.

- Telemedicine enabled maintenance of outpatient care while limiting direct patient contact.

- Fewer than half of the participating countries were prepared for such an event.

What do the new findings imply?

- Providers of NBS service should develop and test contingency plans for prevention of service disruptions during such events in the future.

examinations and supply of laboratory equipment and reagents. Trained staff were sometimes moved to other duties, including intensive care facilities. The pandemic also showed the need to introduce alternative forms of communication with the patients. ${ }^{5}{ }^{6}$ Not all countries had well-developed contingency plans to respond to these events in an organised way.

Neonatal detection and treatment of some congenital disorders are crucial to prevent severe health-related consequences. ${ }^{7-9}$ In response, most developed countries have introduced national newborn screening (NBS) programmes to provide early asymptomatic recognition of a defined panel of disorders where early treatment is effective. This screening is also becoming available in many developing regions in the world. ${ }^{10} 11$ Some recent reports exist about the impact of COVID-19 on diagnosis and management of patients with inborn errors of metabolism included in the NBS programmes. ${ }^{12-14}$ However, little data exist on the overall impact of COVID-19 on NBS programmes. 
To explore the impact-indirect or direct effectof the pandemic on the execution of NBS services, we conducted an international survey. Our main aim was to gather the experiences of the COVID-19 pandemic (first and second waves) from a large and representative number of NBS centres worldwide.

\section{METHODS}

The survey was conducted by inviting professionals who are members of the International Society for Neonatal Screening (ISNS) and are responsible for NBS programmes. The questionnaire was designed by the authors and approved by and distributed with support of the ISNS. The distribution and collection of the questionnaires took place in February and March 2021. Final clarifications and data authorisation were conducted by email.

The questionnaire contained 24 questions, starting with contact details of the participant. In the first part of the questionnaire, participants were asked to evaluate the severity of COVID-19 pandemic in their country/region/ autonomous province and to provide general information about their NBS programmes. The second part contained questions about any impairment in the NBS programmes experienced due to the pandemic, including obstacles to the conduct of testing, confirmatory diagnostics, communication with the parents, possible missed cases, consequent metabolic crises and existing plans/ guidelines for screening in the event of pandemics and serious disruption to health. All participants were invited to consent to participation in the survey, incorporation in the study and their agreement to receive acknowledgement if published. The full questionnaire is available in online supplemental file 1 . The questionnaire used the SurveyMonkey survey platform (SVMK, San Mateo, California) and was distributed to the participants by the ISNS office via email. The responses were collected through the same platform and through email. A fill-in survey was also created on the request of the professionals who wished to summarise or check the answers with other colleagues from the same centre. Patients or the public were not involved in the design, or conduct, or reporting or dissemination plans of our research. All respondents were requested to provide a response also with regard to inclusion of their data and their participation as group coauthors in case of publication.

Responses were obtained from 46 different screening centres from 39 countries. Most of the screening centres that responded were located in Europe, Asia Pacific, North America and Middle East and North Africa (MENA) regions; no responses were obtained from the ISNS regions of Latin America and Sub-Saharan Africa. Centres or countries with more than one answer were weighted for the analysis. Two participants noted that they did not wish to be part of the analysis and their answers were excluded. In three centres from three different countries, duplicate responses were received.
Data on the total number of newborns in the period from 2015 to 2020 were gathered from the World Population Prospects: 2019 Revision (United Nations, Department of Economic and Social Affairs, Population Division, 2019). The World Population Prospects: 2019 Revision, (available at https://population.un. org/wpp/Download/Standard/Fertility/). Data on the number of deaths and COVID-19 cases were gathered from the 'Worldometers' website (available at https:/ www.worldometers.info/coronavirus/?utm_campaign= homeAdvegas1?).

\section{RESULTS \\ Global distribution and characteristics of NBS centres}

Forty-four centres from 38 countries were included in the final analysis. Most of the screening centres that responded were located in Europe (54.5\%); majority of the countries providing responses were also from Europe $(57.9 \%)$. Eight centres $(18.2 \%)$ representing seven countries $(18.4 \%)$ originated from within the Asia Pacific region and four centres $(9.1 \%)$ from two countries $(5.3 \%)$ came from North America. The MENA region submitted responses from five centres $(11.4 \%)$ from five countries $(13.2 \%)$, while three centres $(6.8 \%)$ from two countries $(5.3 \%)$ in the Oceania region were included (tables 1A-E). Unfortunately, no responses were obtained from the ISNS regions of Latin America and Sub-Saharan Africa. For most countries, only one centre responded, while for seven countries responses were received from two centres.

The total number of children screened per year by the participating centres was 3.801 million. The median number of screened children per centre was 40000 (IQR: 9500-85 000). The number of children screened per country ranged from 0 to 950000 (tables 1A-E). Most participating centres (31.8\%) screened for 21-30 diseases, $25.0 \%$ of the centres screened for $1-5$ diseases, $18.2 \%$ screened for $11-20$ diseases, $15.9 \%$ screened for $31+$ diseases, and $6.8 \%$ screened for $6-10$ diseases. One participating centre $(2.3 \%)$ did not screen for any disease (see tables 1A-E).

\section{Impact of the COVID-19 pandemic on countries and NBS centres}

The number of COVID-19 cases per million until 2 May 2021 ranged from 30 to 155000 per million per country (average: $57300 \pm 437$ per million per country). The number of deaths per million due to COVID-19 ranged from 0.5 to 2884 per million per country (average: $1058 \pm 887$ per million per country) (tables $1 \mathrm{~A}-\mathrm{E}$ ). While most of the 35 countries included $(65.7 \%)$ were affected to a greater extent by the second wave of the COVID-19 pandemic, $17.1 \%$ of the countries reported to be more affected during the first wave of the COVID-19 pandemic and $17.1 \%$ were affected equally in both waves of the COVID-19 pandemic. 


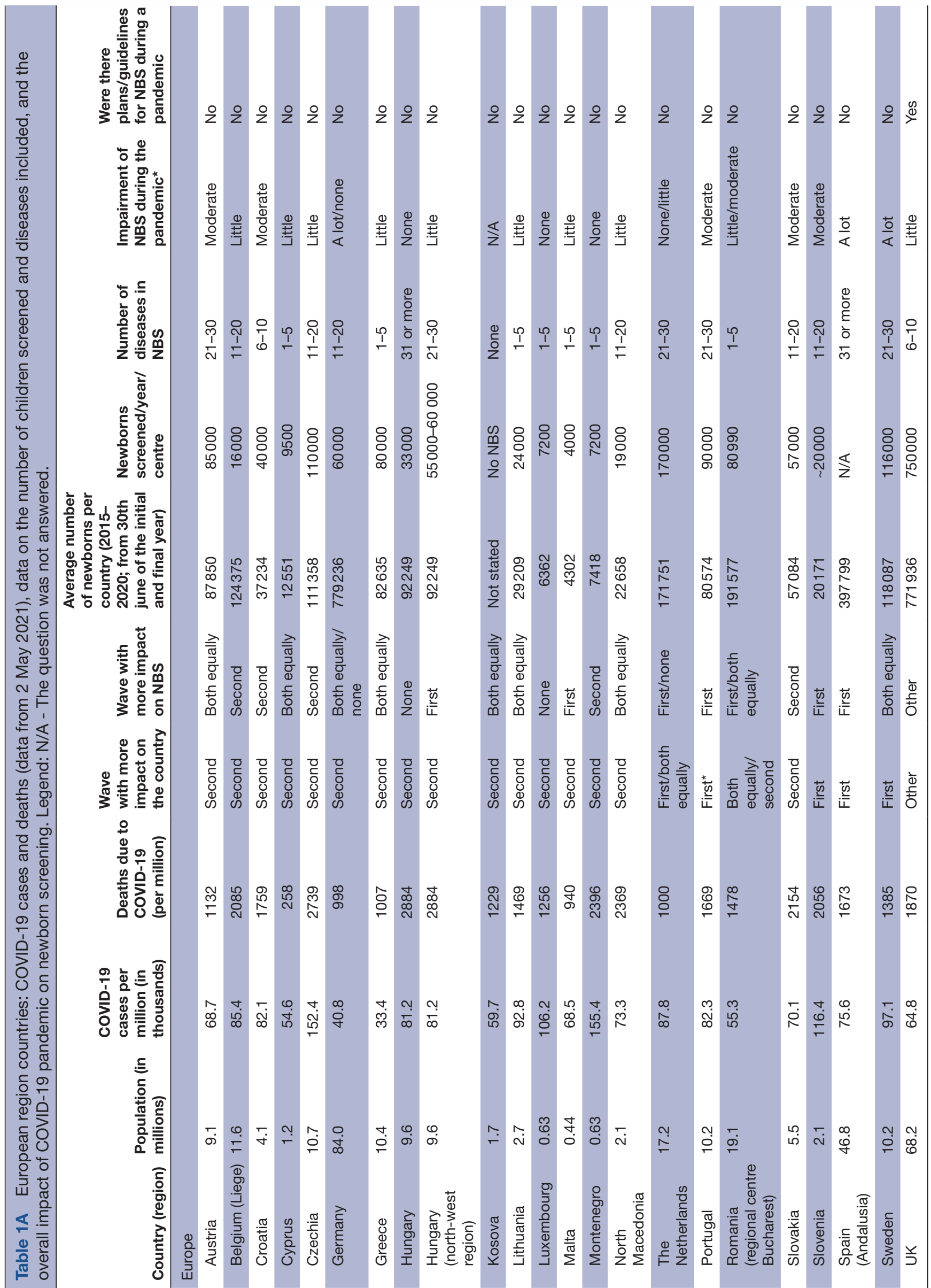



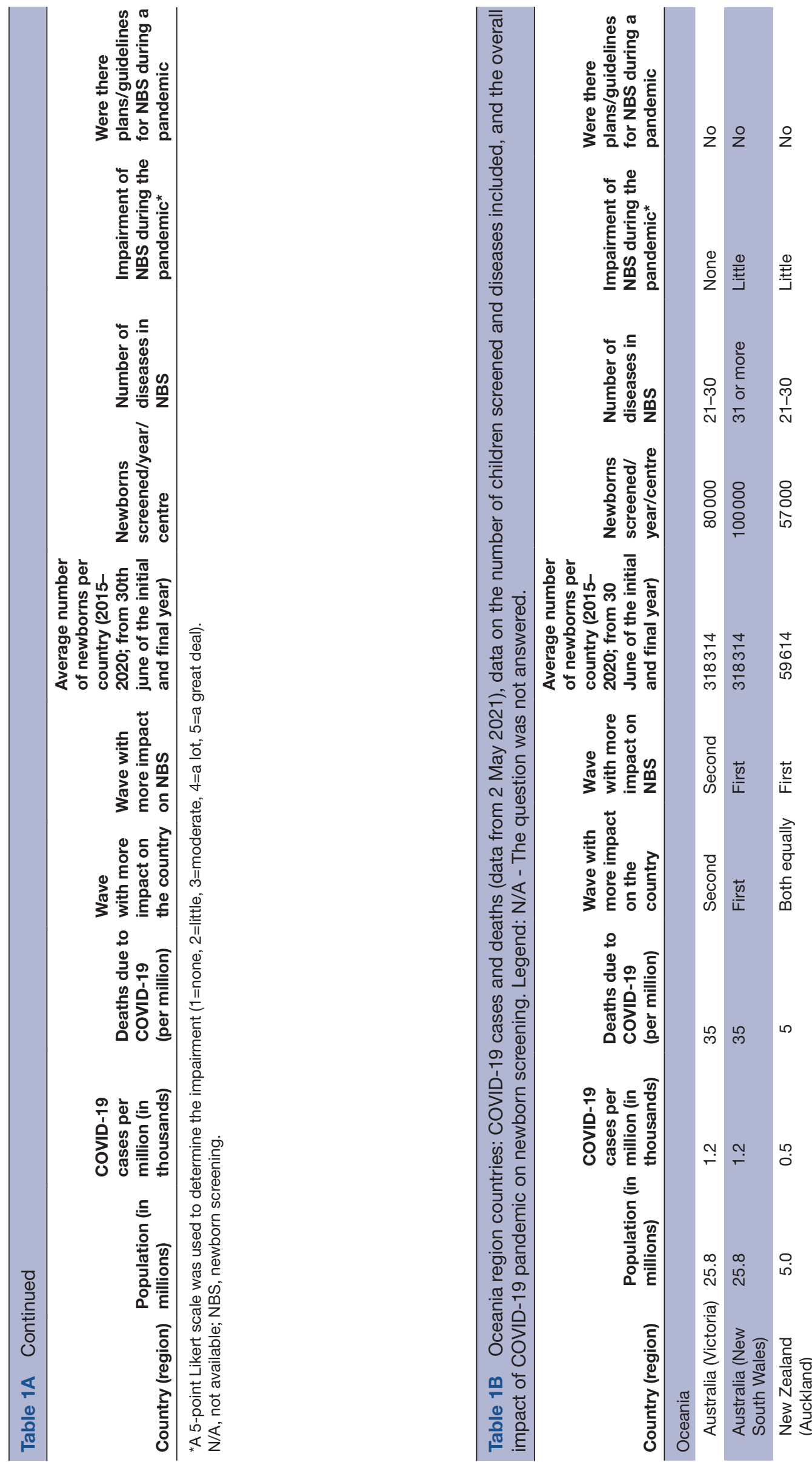

$\div 5$

ะั \$

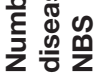

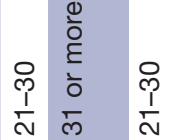

옹 홓

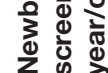

응

ఫे ఫ

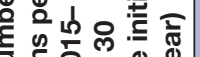

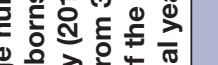

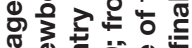

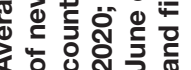

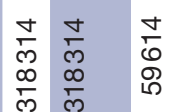

을 울

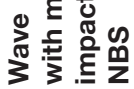

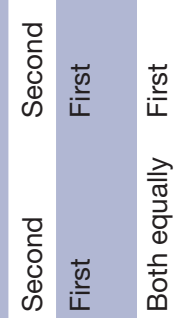

秀雚

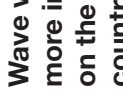

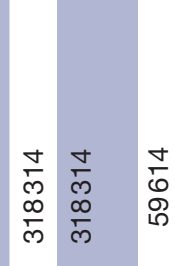

क

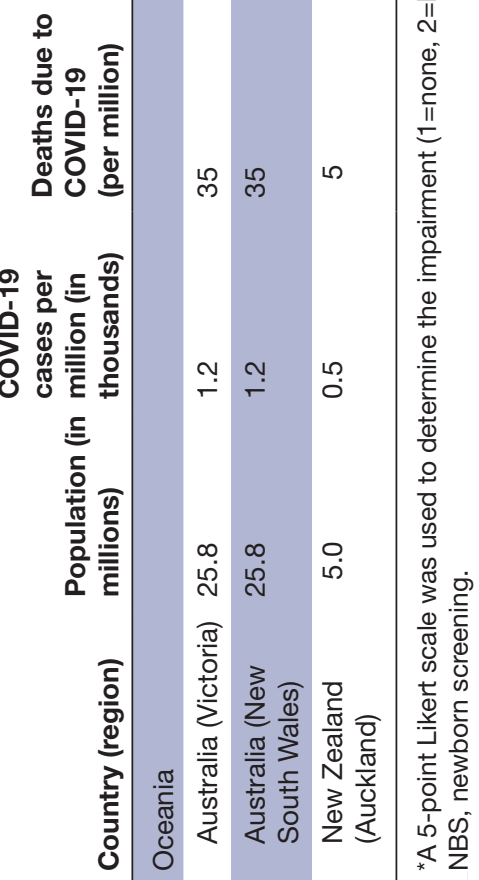




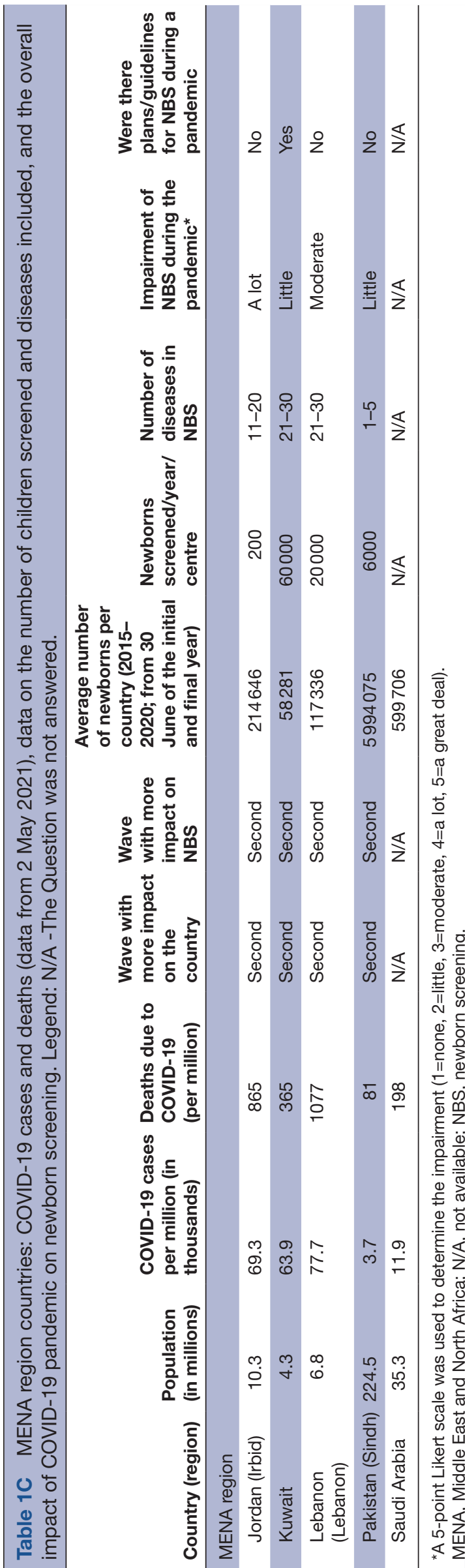

The degree of impact of the COVID-19 pandemic on regions in general and on NBS programmes is presented in figure 1 .

No participating country was unaffected by the COVID-19 pandemic; $33.8 \%$ reported that they were affected a great deal, $28.4 \%$ were affected a lot, $28.4 \%$ were affected to a moderate extent, and $9.5 \%$ reported they were affected only a little.

NBS centres were affected to a lesser extent than the countries as whole. No participating centre reported that they were affected a great deal. Most of the centres responded that the pandemic had created little effect $(47.7 \%), 18.6 \%$ a moderate effect and $12.8 \%$ a lot, while $16.3 \%$ of the centres reported no effect.

Interestingly, despite the higher number of COVID-19 cases in the second wave, NBS centres were reported to be more affected during the first wave of COVID-19 (34.5\%), with $27.4 \%$ of the centres affected equally in both waves and $23.8 \%$ of the centres reporting they were affected more in the second wave of the COVID-19 pandemic and $11.9 \%$ of centres reporting no effect (tables 1A-E).

Only $8.6 \%$ of the participating countries had plans for performing NBS during a pandemic before COVID19. However, the number of countries with plans for performing NBS during a pandemic had increased, with an eventual $38.6 \%$ of the participating countries developing plans. Still, at the time of the survey, $61.4 \%$ of the participating countries did not have plans on how to maintain NBS services during a pandemic (tables 1A-E).

\section{Main issues encountered during the COVID-19 pandemic}

Various obstacles and problems in conducting NBS during the COVID-19 pandemic were encountered (figure 2 and box 1). The main reported obstacles, reported from the most frequent to the least frequent, were lack of staff $(33.8 \%)$, establishing effective communication with parents $(18.9 \%)$, lack of financial support (10.8\%), provision of diagnostic equipment $(8.1 \%)$ and hospital capacity issues related to COVID-19 patient activity $(2.7 \%)$. Of the centres, $25.7 \%$ answered 'other' (fear of contamination, reluctance of parents for COVID-19 testing, transportation problems).

In case of borderline/positive results, informing the family and making an appointment for the family to be seen were moderately affected $(30.4 \%)$, as reported by NBS centres, during the COVID-19 pandemic. Of the NBS centres, $27.1 \%$ reported that these were not affected. Referral of positive children/parents to further diagnostics was sometimes affected in $25.5 \%$ of the NBS centres, while $36.1 \%$ reported no effect.

Many NBS centres $(41.5 \%)$ reported that parents were sometimes reluctant to come to the maternity ward or hospital for retesting/further tests during the COVID-19 pandemic. However, this was variable and $19.5 \%$ of the NBS centres reported that parents were not at all reluctant to come to the maternity ward or hospital for retesting/further tests. 


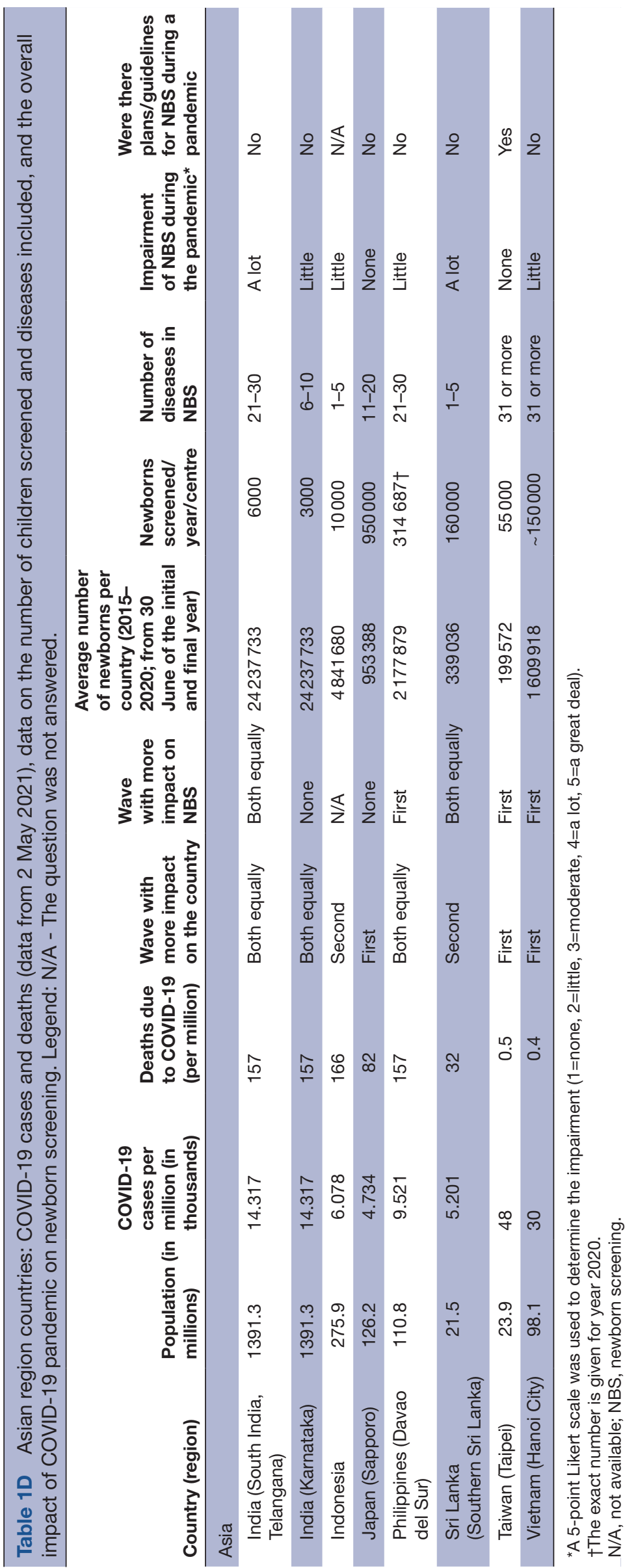




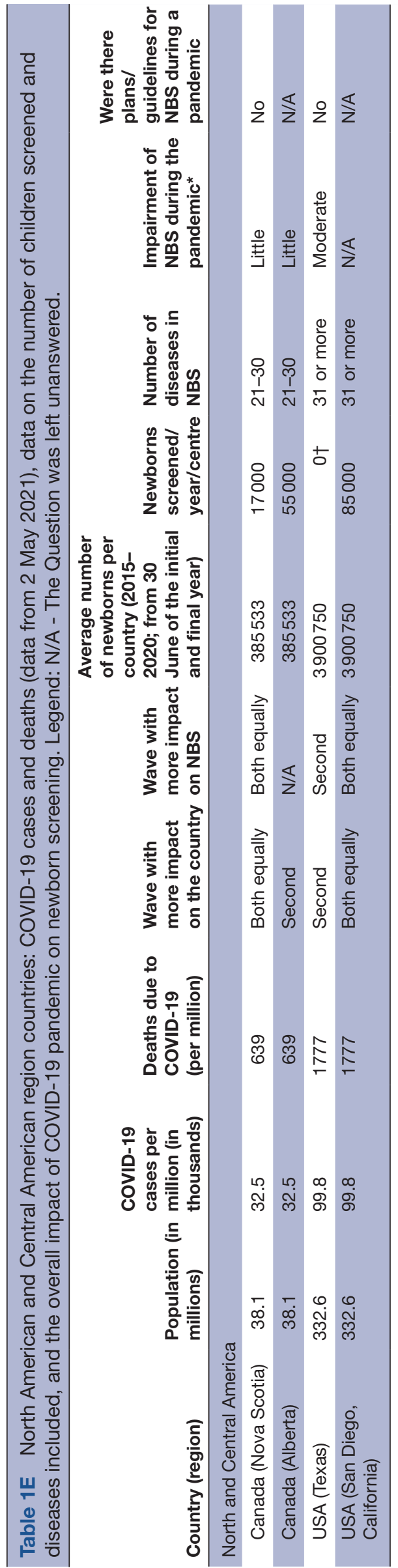

Face-to-face control visits were sometimes substituted by telemedicine during the COVID-19 pandemic. This approach was used by $39.5 \%$ of the NBS centres, while $27.9 \%$ did not implement this approach.

The lack of laboratory and/or clinical personnel responsible for performing NBS during the COVID-19 pandemic was not affected in $22.5 \%$ of the centres. The same number of centres $(32.5 \%)$ reported that the lack of laboratory and clinical personnel had either little or moderate effect on performing NBS during the COVID-19 pandemic. In $12.5 \%$ of the centres, the lack of laboratory and/or clinical personnel had a large effect on performing NBS during the COVID-19 pandemic (figure 2).

Possible solutions suggested (given through the open answer question at the end of the survey; see online supplemental file 1, question 22) to provide NBS services with less disruption through pandemics were establishing virtual consults, NBS centres contacting hospitals or maternity wards or regular virtual meetings, making protocols for sampling in COVID-19-positive mothers, defining the sampling conditions (eg, time of sampling, conditions of posting, etc) and re-emphasising the importance of testing time intervals.

\section{Delays and potentially missed cases during the COVID-19 pandemic}

'No diagnostic delay' due to the COVID-19 pandemic was reported by $33.3 \%$ of the NBS centres. In those who reported a delay in diagnosis, the most frequent response $(24.4 \%)$ was that the delay was only seen occasionally (figure 2).

The most frequent cause of delay in reporting results indicated by the NBS centres was delay in laboratory testing $(36.8 \%)$. As the second most frequent cause of delay, two answers were reported equally (28.9\% each) and indicated either COVID-19 infections in healthcare/ laboratory workers, or COVID-19 contact in the family. Delayed clinical procedure was reported as a cause of diagnostic delay by $26.3 \%$ of the respondents (figure 2).

Delays in the provision of laboratory equipment for NBS during the COVID-19 pandemic were reported by half $(52.4 \%)$ of the participating NBS centres. In those that encountered delays due to provision of laboratory equipment, the delay was mostly seen as moderate (23.8\% of all the answers) (figure 2).

The responses on possible missed cases and metabolic crises are presented in table 2 .

\section{DISCUSSION}

The results of our study showed that COVID-19 impacted the NBS programmes at least partially in most of the responding countries. Majority of the screening centres experienced a broad spectrum of difficulties and were affected to a greater extent in the second wave of the pandemic. Delays and unreliability in the postal service as well as flight cancellations caused delays in sample 


\section{Impact of COVID-19 pandemic on countries and their NBS programs}
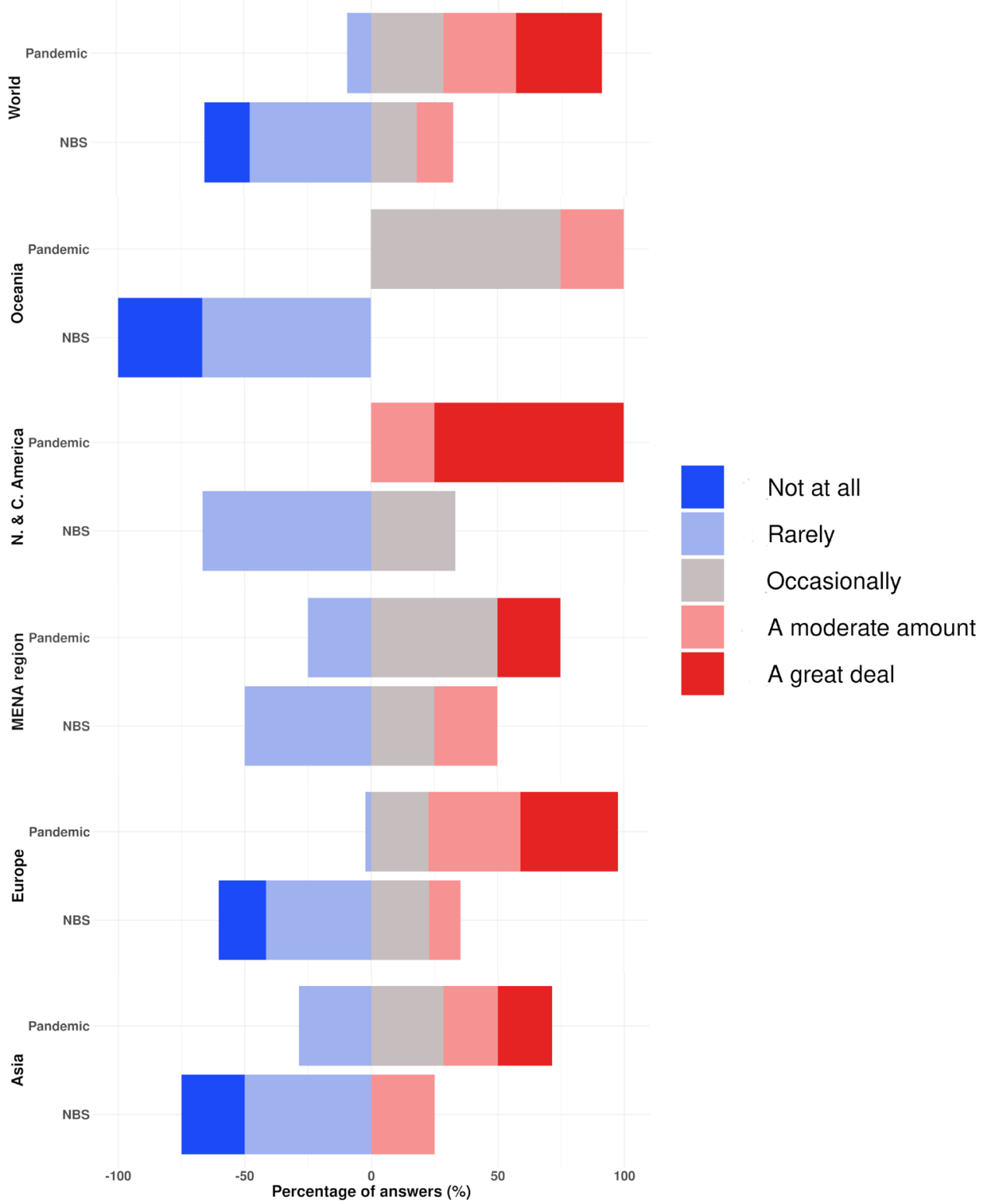

Figure 1 Degree of impact of the COVID-19 pandemic on regions in general ('pandemic') and on newborn screening programmes ('NBS'). MENA, Middle East and North Africa.

transport to screening centres. The provision of laboratory equipment and reagent supplies was also affected. The number of staff available to perform screening was reduced due to infection, quarantine or reassignment to COVID-19 wards, sometimes resulting in complete closure of screening laboratories and the need to send the samples to other centres for analysis.

The collection of samples at home, second-tier tests and follow-up testing were also affected due to parents' fear of infection, social restrictions and effects on public transport. The attention of public health systems generally shifted from provision of NBS services to COVID19-related care. Only a few centres managed to retain a fully functioning NBS programme or had well-developed contingency plans to maintain services during a pandemic.

In general, NBS has been less severely affected by COVID-19 than some other aspects of healthcare delivery, but there are differences among continents (figure 1). For example, Australia, New Zealand, Taiwan and Vietnam had the lowest number of COVID-19 cases per million in the Asia Pacific region and reported little or no impairment of NBS during the pandemic. The first three of these have well-established expanded metabolic screening with tandem mass spectrometry (MS/MS) and NBS with good coverage, all government-funded. Conversely, India reported a significant impact on NBS delivery. The organisation and delivery of NBS in India 


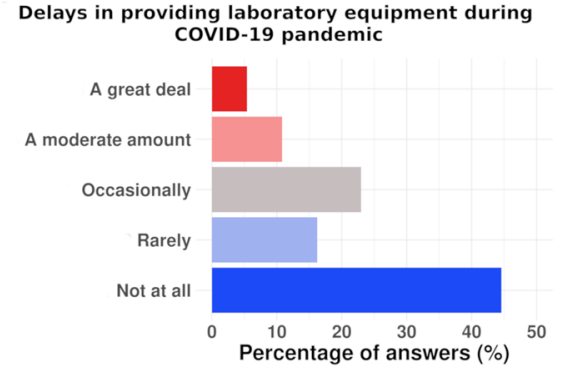

Diagnostic delays during COVID-19 pandemic

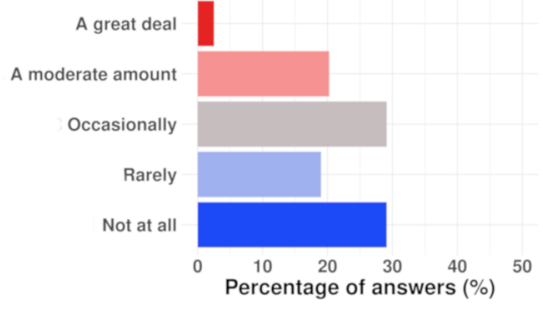

Impact of COVID-19 pandemic on informing parents borderline/positive results

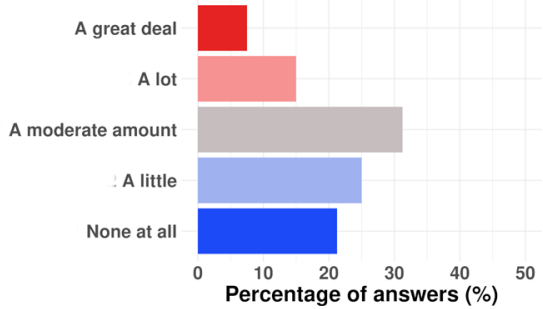

Parents being reluctant to come for retesting/further testing due to COVID-19 pandemic

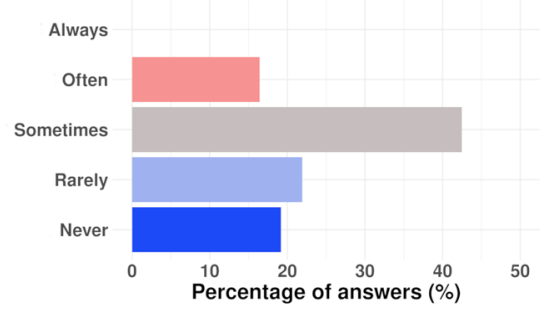

Impact of COVID-19 pandemic on substituting face-to-face visits with telemedicine

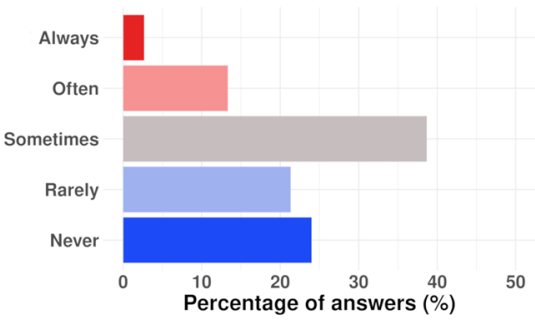

Impact of COVID-19 pandemic on referral of positive children/parents to further diagnostics

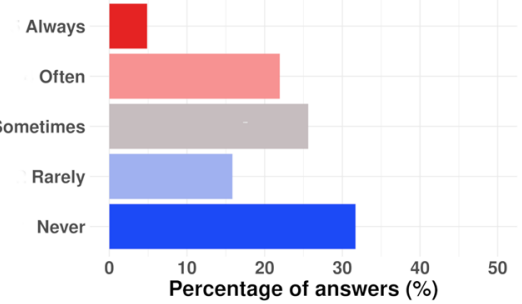

Figure 2 Degree of impact of the COVID-19 pandemic on main aspects of newborn screening programmes.

are less well established, with regional organisation and coverage extending to only $1 \%$ of the population. It is common that the costs of NBS are covered by the family. This was also the country with the most reported COVID-19 deaths per million. ${ }^{11}$

Interestingly, centres that reported their NBS programmes were significantly affected by COVID-19 came from both developed and developing countries. A screening centre in Germany (Berlin) reported disruption due to lack of staff and a regional laboratory was closed due to infection among personnel. Spain reported delays in specimen collection and establishing

Box 1 Main problems encountered in newborn screening (NBS) during the COVID-19 pandemic

Delays and unreliability of postal services.

- Unavailability of new NBS kits and other laboratory equipment.

- Bad sample quality (more births out of hospital, prior hospital discharge, collected outside of the recommended collection time window).

- Staff reduction (due to infection, relocation or exhaustion).

- Blood was not taken in children from COVID-19-positive mothers (early discharge, fear of staff infection).

- Closure of clinics (transformation to COVID-19 clinics).

- Worse communication with field NBS teams.

- Fear of infection among parents visiting hospitals or fear of midwives and general practitioners visiting patients at home. communication with parents due to a restricted postal service during lockdown. Sweden reported problems with travel of overseas technicians needed to maintain laboratory equipment. The NBS centre from southern India reported problems with lack of staff due to disruptions in transport facilities as well as reduced referrals. Jordan and Sri Lanka listed lack of financial support as the biggest obstacle to conducting NBS normally during the pandemic. The clinic in Jordan was closed and the NBS centre in Sri Lanka had problems with sample delivery as well as reagent supply due to airport closure.

The long-term effects of the pandemic on the wellbeing of children with disorders typically diagnosed by NBS remain to be reported.

Delayed laboratory and radiological investigations, interrupted or delayed external technical support for instruments, and interrupted or delayed supply of specialised chemicals, kits and consumables were previously reported. ${ }^{12}$

The proportion of affected personnel, either infected/ in social isolation or taking a leave of absence to take care of family members, varied widely, which is indicative of the varying effects of the pandemic in different countries, regions or cities and of differences in national or local policies in place in different centres. ${ }^{12}$ The fear of staff becoming infected through dried blood spot (DBS) appeared in the beginning of the pandemic, when there was still a lot of unknown about the virus. Although 
Table 2 Presence of missed cases screened by NBS or presence of metabolic crisis in undiagnosed newborns due to COVID-19 pandemic, per centre

\begin{tabular}{llccc}
\hline & Definitely & Probably & Probably not & Definitely not \\
\hline $\begin{array}{llcl}\text { Possible missed cases } \\
\text { Europe }\end{array}$ & $0(0)$ & $4.5(20.5)$ & $14.5(65.9)$ & $3(13.6)$ \\
\hline Oceania & $0(0)$ & $0(0)$ & $2(100)$ & $0(0)$ \\
MENA & $0(0)$ & $2(50.0)$ & $1(25.0)$ & $1(25.0)$ \\
Asia & $1(12.5)$ & $5(62.5)$ & $0(0)$ & $2(25.0)$ \\
North and Central America & $0(0)$ & $0(0)$ & $3(100)$ & $0(0)$ \\
World & $1(2.6)$ & $11.5(29.5)$ & $20.5(52.7)$ & $6(15.4)$ \\
Possible cases of metabolic crisis in undiagnosed newborns & & & \\
Europe & $0(0)$ & $2(8.7)$ & $8.5(37.0)$ & $12.5(54.3)$ \\
Oceania & $0(0)$ & $0(0)$ & $0(0)$ & $3(100)$ \\
\hline MENA & $0(0)$ & $2(50.0)$ & $1(25.0)$ & $1(25.0)$ \\
Asia & $1(12.5)$ & $3(37.5)$ & $3(37.5)$ & $1(12.5)$ \\
\hline North and Central America & $1(33.3)$ & $0(0)$ & $2(66.7)$ & $0(0)$ \\
World & $2(4.9)$ & $7(17.1)$ & $14.5(35.4)$ & $17.5(40.7)$ \\
\hline
\end{tabular}

Data are presented as absolute number (\%).

MENA, Middle East and North Africa; NBS, newborn screening.

vertical transmission of COVID-19 was highly likely, it occurred in only $3.2 \%$ of cases of maternal infection in the third trimester, without significant consequence to the newborns. ${ }^{15}$ One study showed that prenatal diagnostics were also impacted and the rate of women attending screening tests in the first and second trimesters dropped significantly. ${ }^{16}$

Telemedicine emerged as an important tool for maintaining outpatient care while limiting direct patient contact. ${ }^{17}$ It was used for monitoring and follow-up and to increase adherence to medication. ${ }^{18}$ Phone calls and online communication platforms were used to schedule regular meetings to arrange care and provide advice to patients with inborn errors of metabolism (IEM) in $69 \%$ of the centres in the survey. Three have used it even before the pandemic. ${ }^{12}$ Another survey on provider perspectives on the impact of the COVID-19 pandemic on NBS reported significant expansion of telemedicine use for NBS referrals and replacement of inperson ambulatory metabolism visits by telehealth. Nevertheless, nearly $50 \%$ of the respondents indicated that telemedicine was an unfavourable way to conduct NBS triage. ${ }^{19}$

A similar survey undertaken in 16 IEM centres on the impact of COVID-19 on diagnosis and management of patients with IEM showed quantitative declines in almost all aspects of services provided to patients with IEM, between March 1st-May 31st 2020 compared to the same period in 2019, in all except two centres. There was a decline in the number of patients visiting as well as in the laboratory samples obtained. The number of established new metabolic diagnosis decreased by a median of $80 \%$. Of the new diagnoses, $45 \%$ came from NBS; others were clinically or biochemically based. ${ }^{12}$ Decline in the number of patients with IEM attending clinics was observed and could be a result of limiting the number of patient visits per day, referral to other IEM clinics within the same city or country, or travel restrictions enforced by the government. Travel restrictions, disruptions in public transport services and fear of attending hospital during a pandemic increased the number of missed follow-up visits. $^{120-22}$

An international survey of division and group heads in paediatric gastroenterology practice during the COVID-19 pandemic reported a decrease in clinical and research productivity. Staff productivity was impacted due to childcare and mental fatigue. Suboptimal technology, lack of centralised infrastructure, reimbursement concerns and scheduling issues obstructed the use of telehealth. Suggested strategies to prepare for resurgences or another future pandemic included ensuring a well-developed and well-supported telehealth system, ensuring the availability of personal protective equipment, using triage protocols, COVID-19 screening and testing protocols, as well as improving social distancing measures. Another emphasis was made to ensure provider well-being and continuous medical education. ${ }^{6}$

The NBS laboratory service in Victoria managed to develop and implement a four-stage COVID-19 pandemic business continuity plan shortly after the beginning of the pandemic. The NBS workforce was split into two teams, with one working from home and the other on-site, with a weekly rotation. Reliable home team connectivity was established by virtual private network (VPN) access and laptop distribution. Sample collection time frame switched from $48-72$ hours to $36-47$ hours. The protocol for unsuitable samples was reviewed and simplification of process and paperwork was made. It was also decided to undertake fewer repeat collections 
and invasive procedures for the baby while maintaining quality as well as meeting the national guideline and matching other states. The evaluation of the first 20 weeks showed the two-team model succeeded, with mostly positive responses from the NBS staff. While the burden of COVID-19 in Australia was relatively low compared with other countries, the emergency plans continue to be improved by, for example, automation of DBS card reading and working with major hospital systems to ease data entry and reporting processes. ${ }^{14}$

A group of professionals responsible for NBS from the UK reported another example of good practice (through their response to the survey and personal communication with the authors of this survey). A central liaison team at the national level was linked with 16 screening laboratories in the UK by a regular weekly dialogue. A weekly 'Health Check' for laboratory functioning was completed and returned by laboratories and the findings analysed and reported. In addition, a pre-existing national 'baby tracking' system which charts the birth of babies and their subsequent testing for NBS proved to be of great value and was able to identify any delays in sample receipt or results reporting. Taken together, the regular dialogue with testing centres and the baby tracking helped offer assurance and continuity of care.

The strength of our study was that it summoned the experiences from different screening centres worldwide, sometimes covering more than one centre from the same country. The answers came from the professionals directly responsible for the NBS programmes in their countries. As a limitation, we could not reach representatives from South America and Africa, where NBS programmes are not so well established. Furthermore, we did not assess the impact of the pandemic on older patients with IEM and only assessed the experiences during the first two waves of the pandemic.

\section{CONCLUSIONS}

In conclusion, the COVID-19 pandemic in 2020-2021 had severe global-wide consequences not just at all levels of the healthcare system but also on most other spheres of the society. ${ }^{23}$ The pandemic impacted the NBS programmes in most of the responding countries, but fewer than half of the participating countries were prepared for such an event or had contingency plans in place to maintain their NBS programmes during a prolonged time of crisis. Every country or NBS programme should develop and test, perhaps in a virtual form, contingency plans for the 'usual' list of emergencies, such as fire, flooding, earthquakes, strikes, etc. The pandemic presents additional difficulties, and the experience reported in this study indicates the need to reconsider and extend contingency planning to include regional, national or even global disruption. It may be that professional networking within the NBS community has a role to play in developing these plans, perhaps supported to some extent by organisations such as the ISNS.
As the pandemic might be ongoing and pandemics or other large-scale natural disasters could occur in the future, it is important to learn from the experience offered by COVID-19 by identifying both significant shortcomings and examples of good practice so that future planning guidelines and policies might be developed to protect service provision.

Twitter Tadej Battelino @TBattelino and Urh Groselj @UrhGroselj

Acknowledgements The survey was conducted inviting the professionals within the network of International Society for Neonatal Screening (ISNS), which also approved and distributed the survey.

Collaborators COVID-NBS ISNS global network: Australia: Ronda Greaves (Victorian Clinical Genetics Services, Department of Biochemical Genetics, Victoria, Australia), Rosie Junek (New South Wales Newborn Screening Program, Sydney, Australia); Austria: Maximilian Zeyda (Austrian Newborn Screening, Medical University of Vienna, Vienna, Austria); Belgium: François Boemer (University Hospital Center Liege, Liege, Belgium); Canada: Zaiping Liu (IWK Health Centre, Nova Scotia, Canada), Iveta Sosova (Newborn Metabolic Screening and Biochemical Genetics Laboratory, Alberta Precision Laboratories, Genetics and Genomics, Edmonton, Alberta, Canada); Croatia: Ana Skaricic (University Hospital Center Zagreb, Zagreb, Croatia), Tamara Zigman (University Hospital Center Zagreb, Zagreb, Croatia); Cyprus: Alexia Nicolau (Center for Preventive Paediatrics, Limassol, Cyprus); Marios Vogazianos (Center for Preventive Paediatrics, Limassol, Cyprus); Czech Republic: Petr Chrastina (Department of Pediatrics and Inherited Metabolic Disorders, General University Hospital in Prague and First Faculty of Medicine, Charles University, Prague, Czech Republic); Germany: Oliver Blankenstein (Newborn Screening Laboratory Berlin, Charité - Universitätsmedizin Berlin, Berlin, Germany), Martin Lindner (Screening-Zentrum Hessen, Univ.-Hospital Frankfurt, Germany); Greece: Panagiotis Girginoudis (Institute of Child Health, Athens, Greece), Dimitrios Platis (Institute of Child Health, Athens, Greece); Hungary: Gábor Z Rácz (University of Szeged, Szeged, Hungary), Ildikó Szatmári (Semmelweis University, Budapest, Hungary); India: Radha Rama Devi (Rainbow Children's Hospital, Telangana, India), Akila Prashant (JSS Medical College, JSS Academy of Higher Education \& Research, Karnataka, India); Indonesia: Tri Ratnaningish (Universitas Gadjah Mada, Yogyakarta, Indonesia); Japan: Masaru Fukushi (Sapporo Immuno Diagnostic Laboratory, Sapporo, Japan); Jordan: Najah Obiedat (Jordan University of Science and Technology, Irbid, Jordan); Kosovo: Vjosa Mulliqi Kotori (Pediatric Clinic, University Clinical Center, Pristina, Kosovo); Kuwait: May Rushood AIRushood (Kuwait Medical Genetic Center, Kuwait); Lebanon: Kheisser Issam (Newborn Screening Laboratory, Medical Genetics Unit, Saint Joseph University, Beirut, Lebanon); Lithuania: Jurgita Songailiene (Centre for Medical Genetics, Vilnius University Hospital Santaros Clinics, Faculty of Medicine, Vilnius University, Vilnius, Lithuania); Luxemburg: Patricia Borde (Laboratoire National de Santé, Luxembourg, Luxembourg); Malta: Ian Brincat (Mater Dei Hospital, Msida, Malta); Montenegro: Mira Samardzic (Institute for Sick Children, Podgorica, Montenegro); The Netherlands: Eugenie Dekkers (National Institute for Public Health and the Environment (RIVM), Bilthoven, The Netherlands), Rendelien Verschoof-Puite (National Institute for Public Health and the Environment (RIVM), Bilthoven, The Netherlands); New Zealand: Dianne Webster (LabPlus Auckland City Hospital, Auckland, New Zealand); Republic of North Macedonia: Violeta Anastasovska (Department for Neonatal Screening, University Clinic for Pediatrics, Faculty of Medicine, Ss Cyril and Methodius University, Skopje, Republic of North Macedonia); Pakistan: Hafsa Majid (Biochemical Genetics Laboratory, Aga Khan University, Sindh, Pakistan); Philippines: Conchita Gabayan Abarquez (Newborn Screening Center Mindanao, Southern Philippines Medical Center, Davao del Sur, Philippines); Portugal: Laura Vilarinho (Neonatal Screening, Metabolism \& Genetics Unit, National Institute of Health, Lisbon, Porto, Portugal); Romania: Michaela Iuliana Nanu (National Institute for Mother and Child Health, Bucharest, Romania), Florentina Moldovanu (National Institute for Mother and Child Health, Bucharest, Romania); Saudi Arabia: Suhad Almuaigl (Ministry of Health, Saudi Arabia); Slovakia: Mária Knapková (Children's University Hospital Banská Bystrica, Banská Bystrica, Slovakia); Slovenia: Vanja Cuk (University Children's Hospital, University Medical Centre Ljubljana, Slovenia), Ana Drole Torkar (University Children's Hospital, University Medical Centre Ljubljana, Slovenia; Faculty of Medicine, University of Ljubljana, Slovenia), Dasa Perko (University Children's Hospital, University Medical Centre Ljubljana, Slovenia), Ziga Iztok Remec (University Children's Hospital, University Medical Centre Ljubljana, Slovenia), Barbka Repic Lampret (University Children's Hospital, University Medical Centre Ljubljana, Slovenia), Blanka Ulaga (University Children's Hospital, University Medical Centre Ljubljana, Slovenia), Mojca 
Zerjav Tansek (University Children's Hospital, University Medical Centre Ljubljana, Slovenia; Faculty of Medicine, University of Ljubljana, Slovenia); Spain: Raquel Yahyaoui (Newborn Screening Laboratory of Eastern Andalusia, Málaga Regional University Hospital, IBIMA, Spain); Sri Lanka: Manjula Hettiarachchi (University of Ruhuna, Ruhuna, Sri Lanka); Sweden: Rolf Zetterström (Center for Inherited Metabolic Diseases, Karolinska University Hospital and Department of Molecular Medicine and Surgery, Karolinska Institutet, Stockholm, Sweden); Taiwan: Shu Min Kao (The Chinese Foundation of Health, Taipei, Taiwan); UK: James Bonham (Public Health England, UK); USA: Barbara Ferreira (The Lundquist Institute Harbor, UCLA, California, USA), James B Gibson (Ascension Medical Group, Texas, USA); Vietnam: Luyen Quoc Hai (Newborn Screening Center, Bionet Vietnam JSC, Hanoi City, Vietnam), Thu Mai (Newborn Screening Center, Bionet Vietnam JSC, Hanoi City, Vietnam)

Contributors UG, VK and MM conceptualised and designed the study. JGL, TB and JRB reviewed the suggested design and provided further suggestions. JGL and JRB introduced the survey to the ISNS and invited the collaborators. UG, VK and MM carried out the survey and interpreted the results. UG coordinated and supervised data collection and analysis. VK and MM searched for literature from the field and drafted the initial manuscript. UG helped in writing. JGL and JRB helped with emphasising key points and with language. UG, JGL, TB and JRB critically reviewed the manuscript for important intellectual content. UG is the guarantor for the overall content. The group authors from COVID-NBS ISNS global network provided data and shared their experience from the field. The authors (VK, JGL, MM, JRB, TB, UG) approved the final manuscript as submitted and agree to be accountable for all aspects of the work. All the group authors agreed to be listed in case of publication (as members of COVID-NBS ISNS global network).

Funding This work was partly supported by the Slovenian National Research Agency (grant P3-0343).

Competing interests None declared.

Patient consent for publication Not required.

Ethics approval This study does not involve human participants. An ethical committee approval was not applicable because this was a web-based survey study including only healthcare professionals and no sensitive medical data were included.

Provenance and peer review Not commissioned; externally peer reviewed.

Data availability statement Data are available upon reasonable request.

Supplemental material This content has been supplied by the author(s). It has not been vetted by BMJ Publishing Group Limited (BMJ) and may not have been peer-reviewed. Any opinions or recommendations discussed are solely those of the author(s) and are not endorsed by BMJ. BMJ disclaims all liability and responsibility arising from any reliance placed on the content. Where the content includes any translated material, BMJ does not warrant the accuracy and reliability of the translations (including but not limited to local regulations, clinical guidelines, terminology, drug names and drug dosages), and is not responsible for any error and/or omissions arising from translation and adaptation or otherwise.

Open access This is an open access article distributed in accordance with the Creative Commons Attribution Non Commercial (CC BY-NC 4.0) license, which permits others to distribute, remix, adapt, build upon this work non-commercially, and license their derivative works on different terms, provided the original work is properly cited, appropriate credit is given, any changes made indicated, and the use is non-commercial. See: http://creativecommons.org/licenses/by-nc/4.0/.

ORCID iD

Urh Groselj http://orcid.org/0000-0002-5246-9869

\section{REFERENCES}

1 Timeline: WHO's COVID-19 response. Available: https://www.who. int/emergencies/diseases/novel-coronavirus-2019/interactivetimeline?gclid=CjwKCAjwg4-EBhBwEiwAzYAlsnYiA88ooavcwXS Q52xT4HwUCu4PP1BU7yjfFIQg3tP4L7jxXH2GHxoC3OsQAvD_ BwE\#! [Accessed 20 Apr 2021].

2 WHO coronavirus (COVID-19) Dashboard. Available: https://covid19. who.int/ [Accessed 20 Apr 2021].

3 Iftimie S, López-Azcona AF, Vallverdú I, et al. First and second waves of coronavirus disease-19: a comparative study in hospitalized patients in Reus, Spain. PLoS One 2021;16:e0248029.

4 Du R-H, Liang L-R, Yang C-Q, et al. Predictors of mortality for patients with COVID-19 pneumonia caused by SARS-CoV-2: a prospective cohort study. Eur Respir J 2020;55:2000524.

5 Lanpher B, Brunetti-Pierri N, Lee B. Inborn errors of metabolism: the flux from Mendelian to complex diseases. Nat Rev Genet 2006; 7:449-59.

6 Tam SS, Picoraro JA, Gupta SK, et al. Changes to pediatric gastroenterology practice during the COVID-19 pandemic and lessons learned: an international survey of division and group heads. Gastroenterology 2021;161:1052-5.

7 Wilcken B, Wiley V, Hammond J, et al. Screening newborns for inborn errors of metabolism by tandem mass spectrometry. $N$ Engl $J$ Med 2003;348:2304-12.

8 Centers for Disease Control and Prevention (CDC). CDC grand rounds: newborn screening and improved outcomes. MMWR Morb Mortal Wkly Rep 2012;61:390-3.

9 Harms E, Olgemöller B. Neonatal screening for metabolic and endocrine disorders. Dtsch Arztebl Int 2011;108:11-21.

10 Loeber JG, Platis D, Zetterström RH, et al. Neonatal screening in Europe revisited: an ISNS perspective on the current state and developments since 2010. Int J Neonatal Screen 2021;7:15.

11 Therrell BL, Padilla CD, Loeber JG, et al. Current status of newborn screening worldwide: 2015. Semin Perinatol 2015;39:171-87.

12 Elmonem MA, Belanger-Quintana A, Bordugo A, et al. The impact of COVID-19 pandemic on the diagnosis and management of inborn errors of metabolism: a global perspective. Mol Genet Metab 2020;131:285-8.

13 Association of Public Health Laboratories. Impacts to state newborn screening programs form SARS-CoV-2 pandemic.

14 Greaves RF, Pitt J, McGregor C. Newborn bloodspot screening in the time of COVID-19. Genet Med 2021:1-8.

15 Kotlyar AM, Grechukhina O, Chen A, et al. Vertical transmission of coronavirus disease 2019: a systematic review and meta-analysis. Am J Obstet Gynecol 2021;224:35-53.

16 Ozalp M, Demir O, Akbas H, et al. Effect of COVID-19 pandemic process on prenatal diagnostic procedures. J Matern Fetal Neonatal Med 2021;34:1-6.

17 Adams JG, Walls RM. Supporting the health care workforce during the COVID-19 global epidemic. JAMA 2020;323:1439-40.

18 Kayikcioglu M, Tokgozoglu L, Tuncel OK, et al. Negative impact of COVID-19 pandemic on the lifestyle and management of patients with homozygous familial hypercholesterolemia. J Clin Lipidol 2020;14:751-5.

19 Gold JI, Campbell IM, Ficicioglu C. Provider perspectives on the impact of the COVID-19 pandemic on newborn screening. Int J Neonatal Screen 2021;7:38.

$20 \mathrm{Ge} \mathrm{H}$, Wang X, Yuan X, et al. The epidemiology and clinical information about COVID-19. Eur J Clin Microbiol Infect Dis 2020;39:1011-9.

21 Clayton PT. Is susceptibility to severe COVID-19 disease an inborn error of metabolism? J Inherit Metab Dis 2020;43:906-7.

22 Sechi A, Macor D, Valent S, et al. Impact of COVID-19 related healthcare crisis on treatments for patients with lysosomal storage disorders, the first Italian experience. Mol Genet Metab 2020;130:170-1.

23 Osterrieder A, Cuman G, Pan-Ngum W, et al. Economic and social impacts of COVID-19 and public health measures: results from an anonymous online survey in Thailand, Malaysia, the UK, Italy and Slovenia. BMJ Open 2021;11:e046863. 